\title{
New Features of Receptive Fields in Mouse Retina through Spike-triggered Covariance
}

\author{
Jungryul Ahn ${ }^{1}$, Bodo Rueckauer ${ }^{2}$, Yongseok Yoo $^{3 *}$ and Yong Sook Goo ${ }^{1 *}$ \\ ${ }^{1}$ Department of Physiology, Chungbuk National University School of Medicine, Cheongju 28644, Korea, \\ ${ }^{2}$ Institute of Neuroinformatics, ETH Zurich and University of Zurich, Zurich 8057, Switzerland, \\ ${ }^{3}$ Department of Electronics Engineering, Incheon National University, Incheon 22012, Korea
}

Retinal ganglion cells (RGCs) encode various spatiotemporal features of visual information into spiking patterns. The receptive field (RF) of each RGC is usually calculated by spike-triggered average (STA), which is fast and easy to understand, but limited to simple and unimodal RFs. As an alternative, spike-triggered covariance (STC) has been proposed to characterize more complex patterns in RFs. This study compares STA and STC for the characterization of RFs and demonstrates that STC has an advantage over STA for identifying novel spatiotemporal features of RFs in mouse RGCs. We first classified mouse RGCs into ON, OFF, and ON/OFF cells according to their response to full-field light stimulus, and then investigated the spatiotemporal patterns of RFs with random checkerboard stimulation, using both STA and STC analysis. We propose five sub-types (T1-T5) in the STC of mouse RGCs together with their physiological implications. In particular, the relatively slow biphasic pattern (T1) could be related to excitatory inputs from bipolar cells. The transient biphasic pattern (T2) allows one to characterize complex patterns in RFs of ON/OFF cells. The other patterns (T3-T5), which are contrasting, alternating, and monophasic patterns, could be related to inhibitory inputs from amacrine cells. Thus, combining STA and STC and considering the proposed sub-types unveil novel characteristics of RFs in the mouse retina and offer a more holistic understanding of the neural coding mechanisms of mouse RGCs.

Key words: Receptive fields (RFs), Retinal ganglion cells (RGCs), Spatiotemporal white noise stimulation, Spike-triggered average (STA), Spiketriggered covariance (STC)

\section{INTRODUCTION}

Visual information is encoded by the retina, in which spiking activities of retinal ganglion cells (RGCs) are conveyed to the brain $[1,2]$. It is well known that RGCs consist of approximately 20 morphological subtypes [3-5] and about 40 physiological subtypes [68 ]. Each type of RGC shows distinct properties regarding its $\mathrm{ON} /$ OFF response [8-10], direction selectivity [11-14], color vision [15,

Received July 28, 2019, Revised February 19, 2020,

Accepted February 19,2020

\footnotetext{
* To whom correspondence should be addressed. Yongseok Yoo, TEL: 82-32-835-8453, FAX: 82-32-835-0774 e-mail:yyoo@inu.ac.kr

Yong Sook Goo, TEL: 82-43-261-2870, FAX: 82-43-272-1603

e-mail:ysgoo@chungbuk.ac.kr
}

16], contrast adaptation [17-19], intrinsic photosensitive response [20], etc. The receptive field (RF) of a retinal ganglion cell (RGC) acts as the basic element of the visual information processing in the retina [21-23]. The temporal and spatial information of RF are important factors in understanding how visual stimulus is encoded by RGC activities [24]. The RF is obtained by averaging the stimulus patterns eliciting the spikes of a cell. The average of these patterns is known as reverse correlation or spike-triggered average (STA) [25]. Several attempts have been made to use STA for investigating various spatiotemporal patterns of RFs [15, 26-31] and their implication on retinal circuits [32-34]. However, since the STA is the average of the spike-triggered stimulus, potentially more complex patterns in the RF features may be missed. Furthermore, STA may not be suitable for identifying certain cell types; for instance, ON/OFF cells respond equally to the on- and offset of a
Copyright $\odot$ Experimental Neurobiology 2020. www.enjournal.org
This is an Open Access article distributed under the terms of the Creative Commons Attribution Non-Commercial License (http://creativecommons.org/licenses/by-nc/4.0) which permits unrestricted non-commercial use, distribution, and reproduction in any medium, provided the original work is properly cited. 
light stimulus, resulting in a non-informative STA.

As a first-order statistic, STA captures only a single feature in stimulus space. STC on the other hand makes use of the second moment to explore directions of differing variance in stimulus space [25]. Other groups have studied the theoretical properties of STC, its generalization to different stimulus distributions [35], and relation to Wiener / Volterra series [36], and demonstrated its benefits in previous studies on the $\mathrm{V} 1$ complex cells in macaques [37] and the $\mathrm{H} 1$ neurons in fly visual cortex [38]. As for the retinal system, STC analysis on RFs has been applied in salamander [3942] and one monkey study [37], but not yet, to the best of our knowledge, to the mouse retina. In this study we therefore apply both STA and STC analysis to the mouse retina, demonstrating, as in the salamander studies, that STC is able to identify novel characteristics of RFs.

\section{MATERIALS AND METHODS}

\section{Retinal preparation}

Male mice at postnatal Day 56 and later (>P56) (C57BL/6J strain; The Jackson Lab., ME, USA) were used for this study $(n=4)$. Animal use protocols were approved by the institutional animal care committee of Chungbuk National University (approval no. CBNUA-1172-18-02). The mice were anesthetized with an intramuscular injection of $30 \mathrm{mg} / \mathrm{kg}$ tiletamine-zolazepam hydroxide (Zoletil 50; Virbac, Sao Paulo, Brazil), $10 \mathrm{mg} / \mathrm{kg}$ of zylazine hydrochloride (Rumpun; Bayer Korea, Seoul, South Korea), and 5,000 IU of heparin sodium (heparin; JW Pharmaceutical Corp., Seoul, South Korea). The detailed procedures for the preparation of ex vivo retinal patches were described in a previous study [43]. Briefly, the eye was enucleated and then the retina was isolated from the sclera and the retinal pigment epithelium (RPE) and cut into a $2 \times 2$ $\mathrm{mm}^{2}$ patch. The retinal patch was prepared under $4.3 \mathrm{nW} / \mathrm{cm}^{2}$ illumination in an artificial cerebrospinal fluid (ACSF) solution (124 $\mathrm{mM}$ of $\mathrm{NaCl}, 10 \mathrm{mM}$ of glucose, $1.15 \mathrm{mM}$ of $\mathrm{KH}_{2} \mathrm{PO}_{4}, 25 \mathrm{mM}$ of $\mathrm{NaHCO}_{3}, 1.15 \mathrm{mM}$ of $\mathrm{MgSO}_{4}, 2.5 \mathrm{mM}$ of $\mathrm{CaCl}_{2}$, and $5 \mathrm{mM}$ of $\mathrm{KCl}$ ) bubbled with $95 \% \mathrm{O}_{2}$ and $5 \% \mathrm{CO}_{2}$ to maintain a pH of 7.3 to 7.4 and a temperature of $32^{\circ} \mathrm{C}$. The isolated retina was mounted on a planar multi-electrode array (MEA) with the RGC layer down and continuously perfused with oxygenated solution (flow rate: $1 \sim 3 \mathrm{~mL} / \mathrm{min}$ ).

\section{Multi-electrode recording system and signal processing}

The data acquisition system (MEA60 system; Multichannel Systems GmbH, Reutlingen, Germany) included a planar 64-channel perforated MEA (60pMEA200/30iR), an amplifier (MEA1060), temperature control units (TC01), data acquisition hardware ( $\mathrm{Mc}_{-}$
Card), and software (Mc_Rack). The MEA contained 64 circular electrodes in an $8 \times 8$ grid layout with electrode diameters of $30 \mu \mathrm{m}$ and inter-electrode distances of $200 \mu \mathrm{m}$. The electrodes were coated with porous titanium nitride (TiN) and embedded in a perforated polyimide foil that facilitates sufficient oxygen and nutrient supply to the retina [44]. Multi-electrode recordings of the retinal activity were obtained from 59 electrodes, excluding one reference electrode and four inactive electrodes, with a bandwidth ranging from 1 to $3,000 \mathrm{~Hz}$ at a gain of 1,200 . The data sampling rate was $25 \mathrm{kHz}$ per electrode. After high-pass filtering the raw waveform of retinal recording at $100 \mathrm{~Hz}, \mathrm{RGC}$ spikes were isolated using a threshold of four times the standard deviation of the background noise. Multiunit activities containing different spike waveforms were separated into individual cell activities by principal component analysis [45], using the spike sorting software (Offline Sort$\mathrm{er}^{\mathrm{TM}}$; Plexon Inc., TX, USA). Subsequent analysis was done using commercial software (NeuroExplorer $^{\mathrm{TM}}$; Nex Technologies, CO, USA) and custom-made Matlab ${ }^{\text {TM }}$ (MathWorks, MA, USA) codes.

\section{Light stimulation}

Visual stimuli were generated by custom-made software, written in Matlab ${ }^{\mathrm{TM}}$ with Psychtoolbox [46, 47] and presented using a digital light processing (DLP) projector (ep7122; Hewlett-Packard, CA, USA) with a maximum resolution of $1,400 \times 1,050$ pixels and a refresh rate of $60 \mathrm{~Hz}$ (Fig. 1A). The visual stimulus was projected onto the photoreceptor layer with a size of $1.7 \times 1.7 \mathrm{~mm}^{2}$ through the water dipping $20 \times$ objective lens (UMPLFLN20XW; Olympus Corp., Tokyo, Japan), with a beamsplitter (32-506; Edmund Optics Inc., NJ, USA) inserted into the light path for monitoring. Light stimuli were attenuated using a neutral density filter (NE220B; Thorlabs Inc., NJ, USA). For a characterization of ON or OFF RGCs, we applied full-field illumination of 50 repeated trials of 4 $\mathrm{s}$ of a white screen followed by $4 \mathrm{~s}$ of a black screen (Fig. 1B). Light intensity $\left(40 \mu \mathrm{W} / \mathrm{cm}^{2}\right)$ corresponding to photopic vision was measured with a calibrated radiometer (ILT-5000; International Light Technologies, MA, USA). To determine RFs of RGCs, we used a spatiotemporal white noise stimulation, temporally updated at a frame rate of $10 \mathrm{~Hz}$ and spatially arranged in a checkerboard layout with a pixel width of $215 \mu \mathrm{m}$. Each pixel was independently modulated according to the Bernoulli distribution with equal probabilities for +1 (white) and -1 (dark) (100\% contrast and a mean intensity of $3.8 \mu \mathrm{W} / \mathrm{cm}^{2}$ ). This white noise stimulation was applied for $15 \mathrm{~min}$. For temporal resolution of checkerboard stimulus, the visual image was updated at a frame rate of $10 \mathrm{~Hz}$. In general, mouse RGC reaches the peak of the cone-mediated response at $10 \mathrm{~Hz}$ [48]. In this study, the mean intensity of each checkerboard stimulus was $3.8 \mu \mathrm{W} / \mathrm{cm}^{2}$ and this light intensity 


\section{A. In-vitro recording of retina}

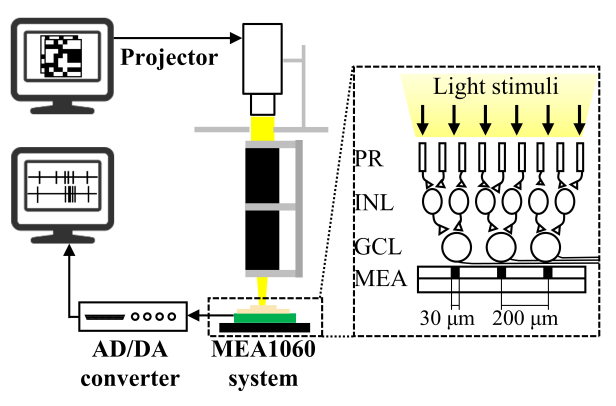

B. Classification of retinal ganglion cells

Full-field illumination

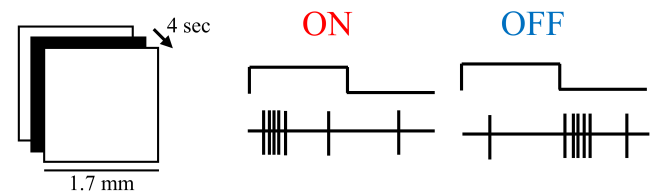

Random checkerboard stimulus

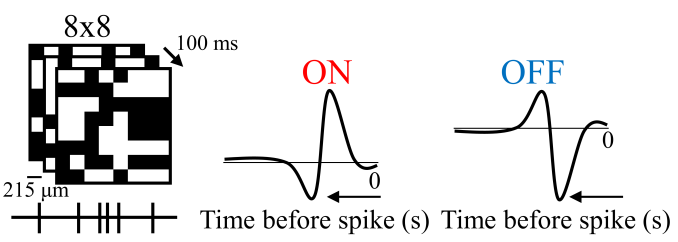

Fig. 1. Light stimulation. (A) invitro recording setup in mouse retina study. Photoreceptor (PR), inner nuclear layer (INL), ganglion cell layer (GCL), multielectrode array (MEA). (B) Functional classification of mouse retinal ganglion cells by full-field illumination and random checkerboard stimulus. corresponded to the photopic vision, which is considered a conemediated response. Thus, temporal resolution of checkerboard stimulus was set to $10 \mathrm{~Hz}$ to obtain the strongest light response of mouse RGCs.

\section{Spike-triggered average and receptive field estimation}

For each light stimulus time bin of $100 \mathrm{~ms}$, spikes from each RGC were counted to produce a train of spike counts at $10 \mathrm{~Hz}$. The STA was obtained by averaging the stimuli preceding a spike with the time window of $900 \mathrm{~ms}$.

(Eq. 1)

$$
\operatorname{STA}(t)=\frac{1}{N} \sum_{n=1}^{N} s\left(t_{n}-t\right) w_{n}
$$

Here, $t_{n}$ is the time of the $n$th spike, $s\left(t_{n}-t\right)$ is the stimulus at the time preceding the spike time $t_{n}$ by $t, w_{n}$ is the number of spikes that occur in the time bin $t_{n}$ corresponding to the $n$th spike, and $N$ is the total number of spikes. A temporal profile of the STA was analyzed for each pixel.

\section{Spike-triggered covariance}

STC analysis is based on the covariance matrix of spike-triggered stimuli:

(Eq. 2)

$$
C=\frac{1}{N-1} \sum_{n=1}^{N}\left[s\left(t_{n}-t\right)-S T A(t)\right]\left[s\left(t_{n}-t\right)-S T A(t)\right]^{T} w_{n},
$$

with the same definitions as in Eq. 1 , and []$^{\mathrm{T}}$ indicating the transpose of the vector. Geometrically, the STC matrix $C$ outlines the ellipsoidal region in stimulus space that elicits a spike response. The extent of this ellipsoid relative to the Gaussian-distributed region of all presented stimuli indicates stimuli that are more or less effective at modulating the cell's firing activity. An eigenvector analysis of the STC matrix $C$ identifies these directions (via eigenvectors) of largest and smallest variance (via eigenvalues). In the following, we use the terms "variance" and "eigenvalues" interchangeably. To identify statistically significant higher or lower variances, a bootstrap with nested hypotheses [25] was performed with 99\% confidence interval. In brief, variances were harvested with randomly shuffled stimuli, therefore these variances indicate the range of variances that can be generated randomly. Then, using 99\% confidence intervals, statistically significant variances were identified and the corresponding eigenvectors further analyzed. To reduce the computational complexity of the bootstrap, a stimulus within $200 \mu \mathrm{m}$ around each MEA electrode was used as the region-of-interest (ROI) for the STC analysis, which actually covers the area of $645 \times 645 \mu^{2}(3 \times 3$ pixels). Considering spatial ( 9 pixels) and temporal (10 frames: -900 to $0 \mathrm{~ms}$ time duration divided by temporal resolution $100 \mathrm{~ms}$ ) dimension, total 90 stimulus dimensions were determined for the STC analysis. Among 90 variances obtained by STC analysis, the smallest variance is always zero because the average (corresponding to the STA) is subtracted out in the covariance calculation (Eq. 2). Thus, we report 89 non-zero variances ordered by their size (also called as 'rank') in eigenvalue spectrum.

Matlab code for processing the experimental data and calculating STA and STC is publicly available at https://github.com/ys7yoo/ retina. For calculating the STC, we followed procedures described by Schwartz et al. [25] and Simoncelli et al. [49] and modified the Pillow lab's STC analysis code available from https://pillowlab. princeton.edu/code_STC.html.

\section{RESULTS}

\section{Typical spatiotemporal profiles of RFs through STA and STC}

A total of 483 RGCs from four mouse retinal patches were used in this study (Fig. 2A). Of these, 358 RGCs (74\%) showed light response to full-field illumination and random checkerboard stimuli. The rest of the RGCs (26\%) showed no light response.

With full-field illumination, functional types of RGCs could be 
A.

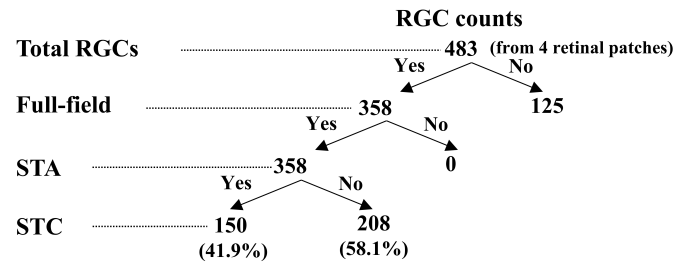

ON RGC

B. Post-stimulus time histogram

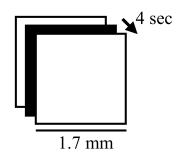

C. Spike-triggered average
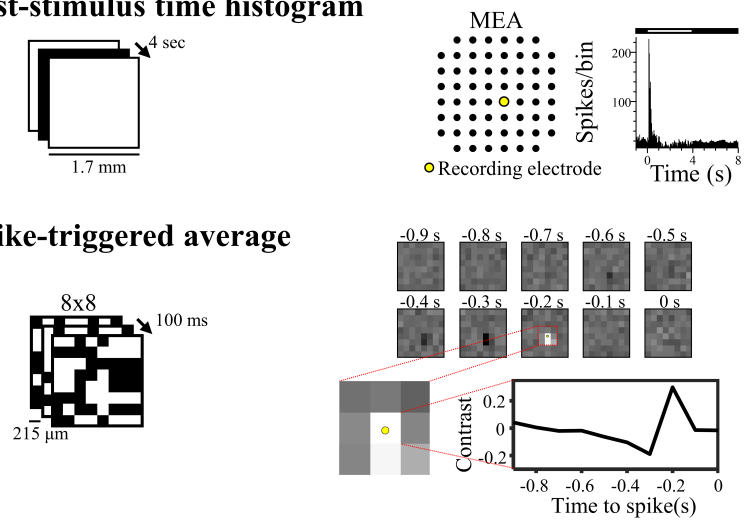

\section{Spike-triggered covariance}

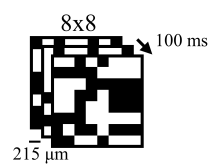

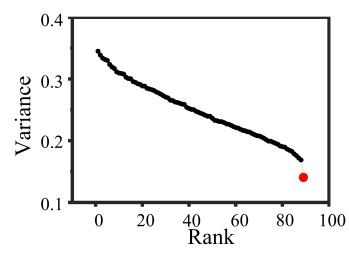

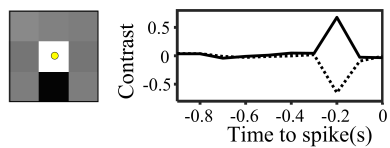

\section{OFF RGC}
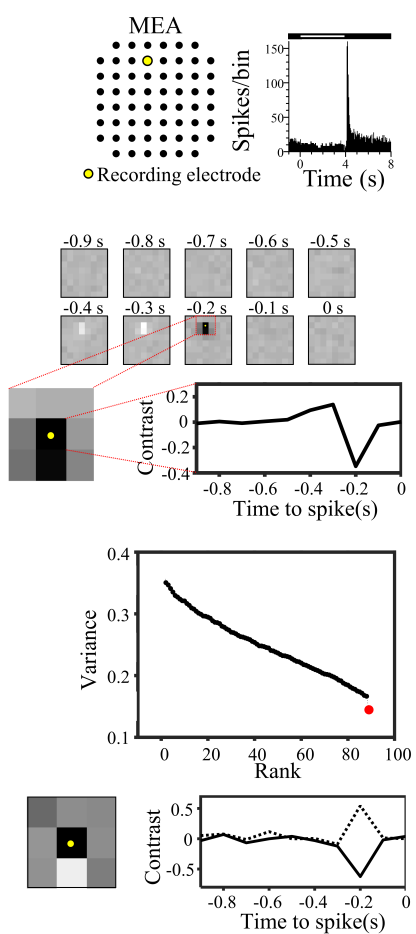

Fig. 2. Typical spatiotemporal profiles of RFs through STA and STC. (A) The number of RGCs used in every stage of the analysis. (B) PSTH graphs of RGC response to full-field illumination of $4 \mathrm{~s} \mathrm{ON}$ and $4 \mathrm{~s}$ OFF duration, respectively (time bin: $50 \mathrm{~ms}$ ). In the $8 \times 8$ grid MEA layout, a yellow circle indicates the recording electrode (ON cell: electrode 55, OFF cell: electrode 42). (C) Upper panel: spatial profiles of STA to a pixel width of $215 \mu \mathrm{m}$ in time from $-0.9 \mathrm{~s}$ to $-0.1 \mathrm{~s}$ before a spike. Bottom left: spatial patterns in ROI of STA marked with a red dotted square consisting of 9 pixels around the recording electrode. Yellow circle indicates the electrode position in the ROI. Bottom right: temporal profile of the one pixel in the $8 \times 8$ grid that shows maximum contrast (right). (D) STC analysis performed in ROI of 9 pixels. Upper panel: eigenvalue spectrum of the STC analysis. Significantly different variances are marked with red dots. Bottom left: Spatial profile of STC $-0.2 \mathrm{~s}$ before a spike. Bottom right: Temporal profile of the two pixels in the ROI with highest absolute contrast (solid line: center pixel; dashed line: bottom center).

classified. Fig. 2B shows post-stimulus time histogram (PSTH) graphs of the RGC response to full-field illumination of $4 \mathrm{~s}$ ON and $4 \mathrm{~s}$ OFF duration. Cells displaying a significant peak at the onset of a flash are considered ON type, cells with a peak at the end of the illumination are OFF RGCs.

For the random checkerboard stimulus, we obtained for all 358 RGCs the averaged stimulus pattern preceding a spike, using STA. In addition, STC analysis revealed statistically significant eigenvector-eigenvalue pairs in 150 RGCs, suggesting that STC provides additional information about RFs for $42 \%$ of the light-sensitive RGCs.

We further investigated these 150 RGCs with statistically significant eigenvector-eigenvalue pairs as follows. From the STA analysis, ON and OFF RGCs were classified. The left panel of Fig. $2 \mathrm{C}$ shows a representative STA of an ON RGC. A spatial map of $8 \times 8$ pixels displays clusters of white pixels with positive contrast change in time. The temporal response at maximum contrast appears about 0.2 seconds preceding a spike. On the other hand, the OFF RGC shows a pattern with a black pixel cluster and negative contrast change (Fig. 2C right). As for the $\mathrm{ON}$ cell, the temporal response at maximum contrast occurs 0.2 seconds before the spike. This spatiotemporal pattern of the STA was evident in the ROI of $3 \times 3$ pixels.

In the STC analysis, significantly lower variances were identified (Fig. 2D, red dot in the top panel), and corresponding eigenvectors were used to search for spatiotemporal patterns in the covariance 
of the spike-triggered stimulus (Fig. 2D, bottom panel). Spatial patterns of the significant eigenvectors exhibit a contrasting pattern between the center pixel and a neighboring pixel, with the temporal profile showing both positive and negative peaks 0.2 seconds before the spike, respectively. The spatiotemporal pattern of STC was clearly different from that of STA, indicating additional features of RFs available through STC.

\section{Temporal pattern classification based on STC}

Temporal patterns of those 150 RGCs with statistically significant variances and associated eigenvectors were further analyzed as follows. Fig. 3 compares STA and STC for the ON (left) and OFF (right) RGCs. In Fig. 3, one RGC (cell \#1) displays the typical temporal pattern of STA. In the STC, one significantly higher and two significantly lower variances (red dots in an STC graph) were identified. The higher variance shows a biphasic temporal profile, the two lower variances a monophasic pattern along the corresponding eigenvector. One of the lower variances shows alternating peaks in the spatial response, represented by spatial patterns with the same polarity at different pixels at different times. The other variance has the same contrasting pattern as in Fig. 2D.

A second representative RGC (cell \#2) has two low variances, one with a monophasic, the other with a biphasic temporal profile like the higher variance of cell \# 1 but a more transient time course.

In the OFF RGC, we can also see contrasting patterns with lower variances, as shown in cell \#1. Cell \#2 includes one higher variance and one lower variance, both with a transient biphasic temporal profile and a contrasting pattern. These various types of temporal patterns have been found in both ON and OFF RGC.

For further understanding of the STC results, we categorized shapes of 205 temporal profiles of 150 RGCs into five types as follows (Fig. 4A): broad biphasic pattern (T1), transient biphasic pattern (T2), contrasting pattern (T3), alternating pattern (T4), and monophasic pattern (T5). Both ON and OFF RGCs show five temporal patterns (Fig. 4B). For both ON and OFF cells, the contrasting pattern (T3) is the most frequently observed (ON: $35.8 \%$, OFF: 55.5\%).

Next, we repeated the type classification separately for higher and lower variances (Fig. 4C). Such a classification is worthwhile for two reasons: (1) It may allow associating a pattern with a RGC type. For instance, T2 can be seen as a signature of ON/OFF cells, as shown in the next section. (2) It sheds light on whether a pattern has a tendency to support or suppress a spike response. For these reasons, we divided the five temporal patterns into higher and lower variances. Biological interpretation on these variances will be introduced in discussion.

The broad biphasic temporal pattern T1 seems to occur at higher variance for both ON and OFF RGCs. It is not clear whether the T2 pattern has the tendency of higher or lower variances. The monophasic groups T3, T4, and T5 tend to coincide with lower variances in both $\mathrm{ON}$ and OFF RGCs.

To summarize the finding in Fig. 4C, we observed a tendency of the biphasic pattern $\mathrm{T} 1$ to occur at higher variance for both $\mathrm{ON}$ and OFF cells, while the monophasic groups T3-T5 occur more often at lower variance in both RGC types. For T2, on the other hand, such a trend cannot be drawn conclusively, which leads us to a more thorough investigation of this pattern in the next section.

\section{T2 temporal pattern in STC as an indicator of $\mathrm{ON} / \mathrm{OFF}$ RGCS}

Unlike the other four temporal patterns, the transient biphasic pattern T2 occurred for both ON and OFF cells at both lower and higher variances (Fig. 4C). One possible explanation is that T2 is in fact associated with neither ON nor OFF, but instead with ON/ OFF cells. To confirm this assumption, we investigated STA and STC based on cell types (ON, OFF, and ON/OFF RGC) by PSTH
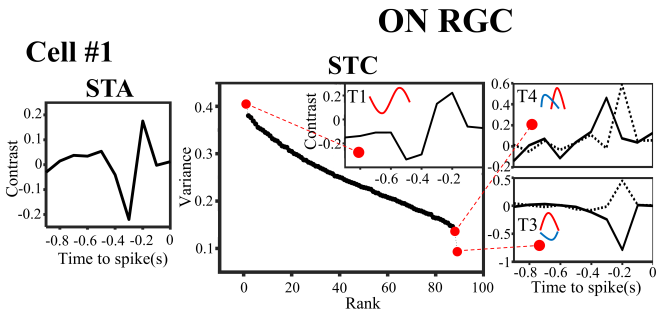

Cell \#2

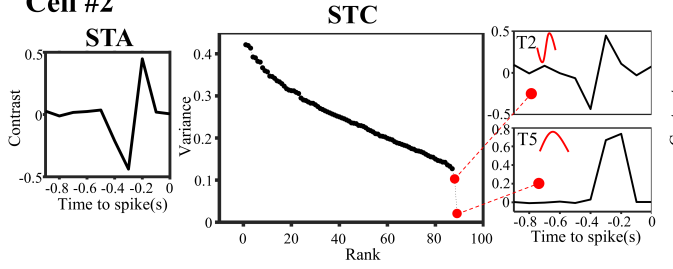

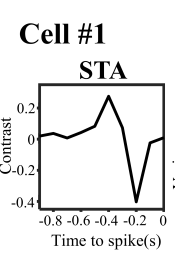

Cell \#2

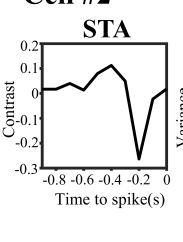

OFF RGC
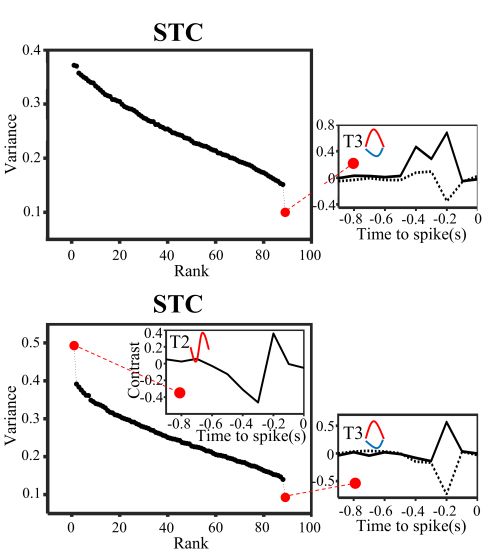

Fig. 3. Exemplary temporal patterns of STC responses found in ON and OFF RGCs. Red dots indicate the significantly higher or lower variances shown in the eigenvalue spectrum. Each temporal profile corresponding to the significant variances is displayed. Five types of temporal profiles are shown in four $(2 \mathrm{ON}$, 2 OFF) representative RGCs. 
A.

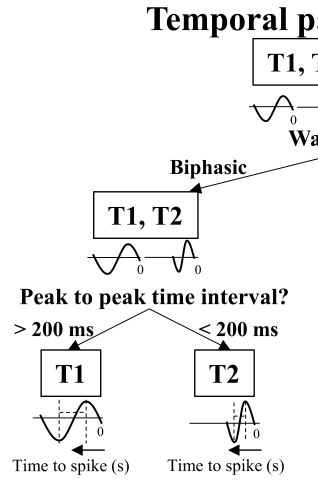

B.

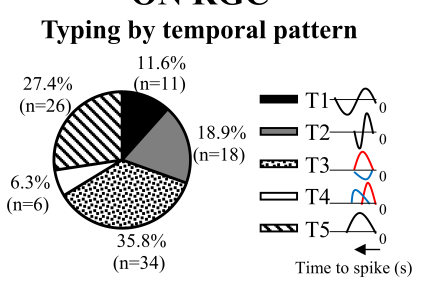

C. Relationship between variance $\&$ temporal pattern

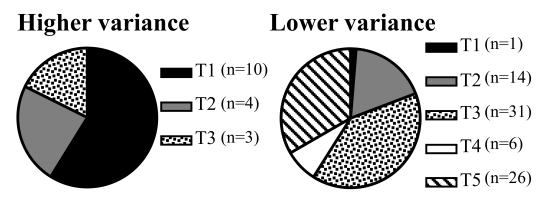

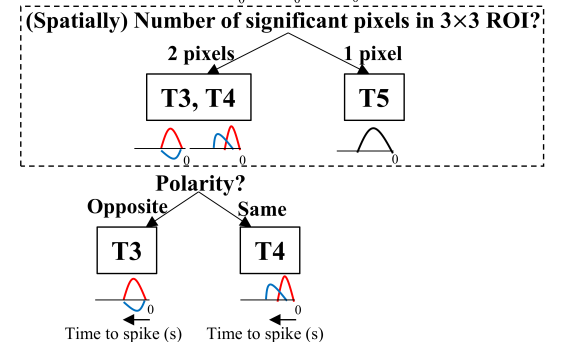

OFF RGC

Typing by temporal pattern

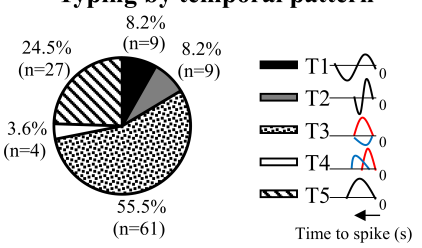

Relationship between variance \& temporal pattern

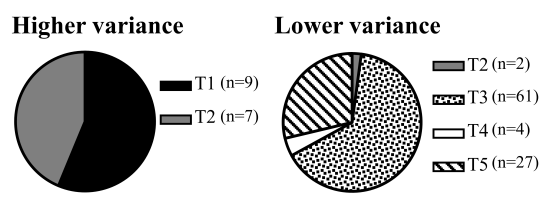

Fig. 4. Temporal pattern classification based on STC analysis. (A) Classification of five temporal patterns (T1-T5) obtained by STC analysis. (B) Left: type distribution of 95 temporal patterns found among the $59 \mathrm{ON}$ cells. Right: distribution of 110 patterns found among the 91 OFF cells. (C) The relationship between significantly higher or lower variances and their respective type of temporal pattern.
(Fig. 5A). The ratio of ON, OFF, ON/OFF RGC cells were about 1 : 1: 2 (22\%: 26\%: 52\%). The STAs of ON and OFF RGCs showed a typical temporal profile with strong peaks as in Fig. 2C. However, the STA of ON/OFF RGCs shows weaker peaks in contrast (middle of Fig. 5B), which makes STA less suited to identify and characterize ON/OFF RGCs.

On the other hand, STCs of ON/OFF cells show distinct patterns. We explored spatiotemporal patterns of STC eigenvectors associated with significantly higher or lower variances (Fig. 5C). Specifically, we determined the five temporal patterns of the higher or lower variances separately for ON, ON/OFF, and OFF cells (Fig. 5D). For lower variance, the temporal patterns T3 and T5 were those most frequently found across all ON, ON/OFF, and OFF RGCs. For higher variance, while temporal patterns T1 appeared across cell types, T2 only appeared in ON/OFF cells and T3 was found only in two ON/OFF cells. This result suggests that temporal pattern $\mathrm{T} 2$ is a strong indicator of ON/OFF RGCs.

\section{DISCUSSION}

\section{New features of RFs provided by STC analysis}

To investigate the RGC RF profile, we performed STA and STC analysis. While STA showed an average RF pattern of RGCs, STC showed additional spatiotemporal patterns not available from STA, which accounts for $42 \%$ of all analyzed cells. No RGCs presented only STC-positive but STA-negative RFs. As suggested earlier [25], this finding confirms that while STA acts as a major component of RF, STC seems to extract additional filters by which the RGCs encode visual stimuli. Sixty percent of RGCs (208/483 cells) did not need STC to fully describe their RF, which seems to indicate that one averaged stimulus filter is enough to process visual stimuli in these cells. On the other hand, $42 \%$ of RGCs had at least two stimulus filters for encoding visual information.

Through our STC analysis, five temporal types were classified as follows: broad biphasic pattern (T1), transient biphasic pattern (T2), contrasting pattern (T3), alternating pattern (T4), and monophasic pattern (T5). T1 has been found to be associated with a high-ranking eigenvalue of the STC matrix (a higher variance along the corresponding direction in stimulus space). As described 


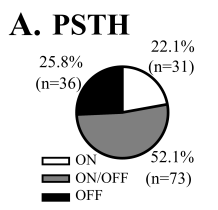

B. STA

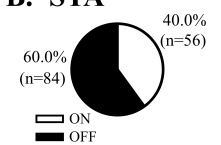

C. STC
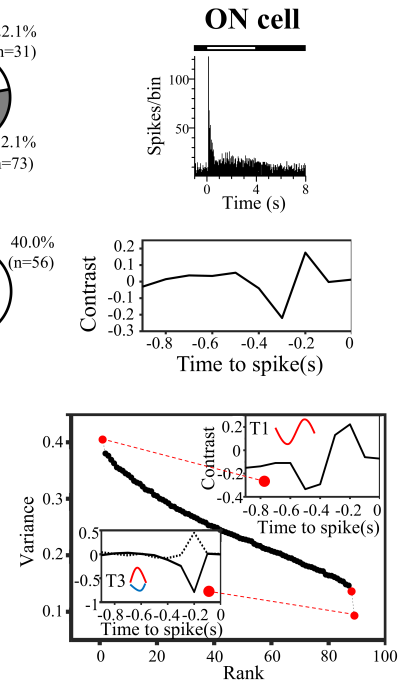

D.

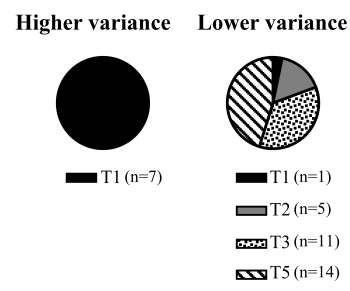

ON/OFF cell
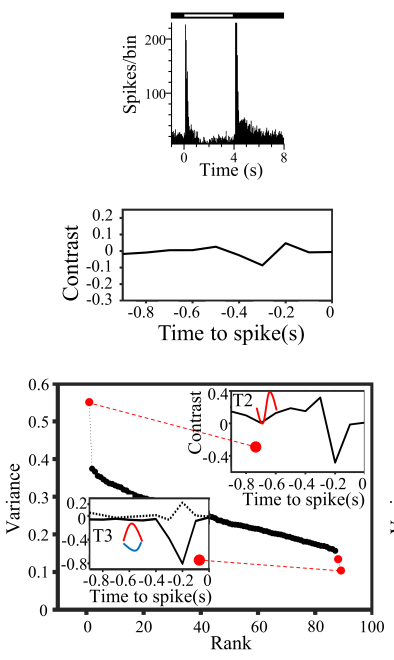

Higher variance Lower variance

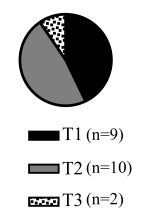

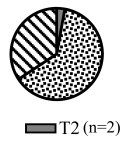

$\mathrm{T} 3(\mathrm{n}=44)$ هT5 (n=24)
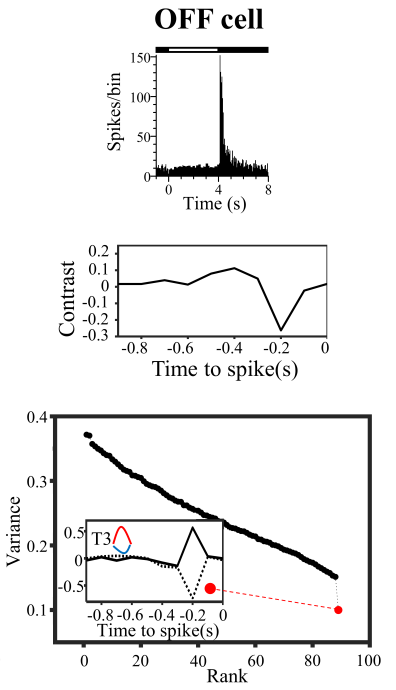

Higher variance Lower variance
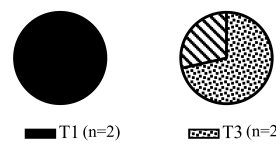

$\rightarrow \mathrm{T} 3(\mathrm{n}=25)$ $\infty \mathrm{T} 5(\mathrm{n}=10)$
Fig. 5. Matching of $\mathrm{ON}, \mathrm{ON} /$ OFF, and OFF RGC with STCbased temporal pattern type. (A) PSTH graphs of RGC response to full-field illumination of 4 s ON and 4 s OFF duration respectively (time bin: $50 \mathrm{~ms}$ ). Three types of ON, ON/OFF, and OFF responses are shown. The percentage of the three types classified through PSTH is shown in the pie chart. (B) STA temporal profiles of $\mathrm{ON}, \mathrm{ON} /$ OFF, and OFF RGCs. (C) STC eigenvalue spectrum for $\mathrm{ON}$, ON/OFF, and OFF cells. The temporal profiles of significant eigenvalues (marked by red dot) are shown as insets. (D) Distribution of the five temporal profiles for significantly higher and lower variances, separately for $\mathrm{ON}$, ON/OFF, and OFF cells. earlier [25], a higher variance indicates an excitatory filter and a lower variance an inhibitory filter for modulating the cell's firing activity. In light of this, $\mathrm{T} 1$ could be originating from bipolar cells which are known as excitatory neurons to RGCs in retinal circuits [50-52]. On the other hand, T3-T5 coincided with lower variances, which could be related with inhibitory filters originating from amacrine cells [53-55]. Finally, T2 showed inconclusive results when considering only ON and OFF RGCs. PSTH analysis indicated that the high-variance T2 pattern is likely to be found in the ON/ OFF cell type. We therefore propose $\mathrm{T} 2$ an indicator of ON/OFF cells.

STA and STC provide complementary information on RFs of RGCs. We focused on what kinds of additional information can be obtained from STC. As an alternative approach, Cantrell et al developed a variant of STC, which they termed as Spike triggered covariance-non centered (STC-NC) to identify ON/OFF cells [56]. Since STA, which is the mean visual stimulus that precedes a spike fails to classify ON/OFF cells, STC analysis which identifies multiple relevant linear filters provides a better analytical approach. However, conventional STC analysis can be cumbersome, therefore, they developed STC-NC analysis which maintains the simplicity of a single filter analysis to characterize different RGC types including ON/OFF cell. STC-NC has advantage of less time consuming which deals only with the highest eigenvalue to count the maximal deviation of the spike-triggered stimuli around zero. Ironically, by dealing with only one filter, STC-NC has disadvantage of not capable of full characterization of the RF properties of RGCs. In our present study, we try to fully characterize the RF properties through STC, not found in STA. Although our STC tool requires a large number of spikes and time consuming for data analysis, multiple filters extracted from the covariance matrix from which STA is removed enabled us to fully characterize diverse RF properties such as T1-T5 temporal patterns.

In this study, we extended STC to explore RFs of mouse RGCs. Previous studies on RGCs are limited to salamander retina, which is the most widely used retinal model for in-vitro MEA study due to the single-layer structure of salamander RGCs. One of the most relevant salamander studies of Fairhall et al's [39] performed similar analysis based on STC. They applied covariance analysis to determine what stimulus features are relevant to each RGC's firing. Their interest was more focused on how much information about stimulus features RGC could convey along the visual pathway to the brain not the receptive field property of each RGC. They showed that covariance analysis was highly successful at describing how RGCs encode temporal patterns of light intensity. However, regardless of different research goals, there is a remarkable similarity with both studies. For their "bimodal cell" which shows two separate clouds in the plane formed by the projections onto 
two visual features are strongly ON/OFF cell in their response to light stimulation (Fig. 9 from [39]). In addition, it was also reported that a significantly higher variance of the 'bimodal cell' is a key factor to lead this bimodal pattern of the ON/OFF cell. The fact matches our finding that ON/OFF RGCs are mainly discovered in T2 pattern with significantly higher variance (Fig. 5). In our study on mouse retina using MEA, we divided RGCs functionally into ON, OFF, and ON/OFF cell types through PSTH patterns using full-field illumination. Then, through STC analysis, we found that a mixture of five temporal patterns is distinctively shown in an individual RGC (Fig. 3-5). Except T2 pattern, an indicator of ON/OFF cell, the rest of all four temporal patterns appeared in individual RGC, irrespective of cell types. Each pattern reflected a fraction of synaptic inputs coming into one RGC; T1 (excitatory synaptic input from bipolar cell), T3-T5 (inhibitory synaptic input from amacrine cell which is well known as an inhibitory neuron in the inner nuclear layer of retina). Through this effort, we identified the complex RF properties in the individual RGC.

\section{Pros and cons of STA and STC analysis}

STA and STC are based on the mean and covariance of spiketriggered stimulus, respectively. Therefore, STA requires lower computational complexity and is easy to interpret. However, STA provides limited information about RFs and fails to convey complex patterns. In contrast, STC requires higher computational complexity and its high-dimensional structure is not straightforward to interpret. However, STC is able to capture rich RGC responses. Therefore, in an effort to address the shortcomings of STC analysis, reducing the dimension of the stimulus space is an active research topic [37]. We reduced the computational complexity by setting up a $3 \times 3 \mathrm{ROI}\left(645 \times 645 \mu \mathrm{m}^{2}\right)$ for STC analysis, which is still large enough to capture important RGC responses considering that the average RF diameter is $200 \mu \mathrm{m}$.

In this study, we focused on what kinds of RGC response patterns are identified by STC but not by STA. Most importantly, STC provides richer characterization of RGC RFs. As shown in Fig. 3 and 4, STC revealed additional spatiotemporal patterns in the RF. These patterns are closely related to the visual encoding process of the RF, in which STC patterns function as stimulus filters needed to encode visual stimulus into spiking information. Since RGC functions as a nonlinear space-time integration module in retinal encoding systems [41], these stimulus filters can be utilized as elements of linear and nonlinearity functions for computational models such as generalized linear models to predict the spiking of RGCs [24].

Therefore, when STA and STC are considered together, the performance of the computational model to predict the spiking rate of RGC will be improved. The synergistic potential of STA plus STC application has already been proven in salamander retina study [39]. In future study, these combined filters can be applied to the modeling study of mammalian retina, which is more complex than the salamander retina. As an alternative to overcoming the drawbacks of STA and STC, a spike-triggered non-negative matrix factorization (STNMF) method was introduced by Liu et al. [57]. It decomposes the ensemble of effective spike-triggered stimuli into multiple subunit modules using non-negative matrix factorization, enabling STNMF to detect the layouts of localized RF subunits. Through STNMF analysis, the previous study has confirmed that each localized subunit represents the presynaptic input of inner retinal neurons to RGC in the salamander retina. Despite lower computational complexity compared with STC and richer characterization of RGC RFs compared with STA, the tool is limited to investigating spatial patterns of RFs, not temporal patterns.

\section{Future work \\ Stimulus design}

In this study, we designed a stimulus protocol with random binary checkerboard for spatiotemporal RF patterns of RGCs. Binary checkerboard stimuli have been gold standard stimulus for finding $\mathrm{RF}$ in retina $[23,24,26,28,29]$. Recently, correlated noise stimuli is used to distinguish ON and OFF RFs from ON/OFF cells [58]. In future work, we aim to apply these stimulus paradigms together with STC analysis to identify functional RGC types.

Other studies regarding visual cortex suggested more advanced stimulus techniques to better reconstruct RFs of cortical neurons. For instance, an oriented wavelet stimulus was used to find the orientation selectivity of neurons [59], or 2D noise stimuli to reveal the diverse RFs properties of cortex neurons such as complex cells [60]. These stimulus techniques could be incorporated to our experiment to differentiate orientation selective RGCs.

\section{Applications}

STA and STC techniques can be applied to vision research for retinal diseases as well as normal vision. In particular, RF changes in RGC have been studied using STA in glaucoma mouse models with progressive degeneration of retinal function [61, 62]. Retinal diseases caused by photoreceptor loss such as age-related macular degeneration and retinitis pigmentosa induce remodeling of the retinal circuitry and physiological changes in RGCs [63-67]. We assume that the RF of RGC may change as retinal degeneration progresses [68].

Electrical stimulation of surviving retinal neurons is known as a prominent strategy for restoring blind vision $[69,70]$. Recently, the development of biomimetic electric pulse protocols has been stud- 
ied by many research groups [71-73]. A prerequisite of the task is to find an electric pulse that can reproduce the physiological light response. Like RGC response to white noise stimulation, electrical pulses with Gaussian white noise were used for retinal stimulation and the electrical receptive field (eRF) of RGC was reconstructed using STA [74-76]. In addition to previous studies using only STA, Maturana et al. [77] evaluated the eRF of RGC through not only STA but also STC analysis, which revealed several stimulus filters. Thus, STC analysis provides useful information about how RGCs encode complex visual or electrical inputs.

Future work in this direction includes applying STA and STC analysis to determine the RF pattern change of the degenerated retina compared to the normal retina, and how the visual information encoded by RGCs varies with the degeneration stage.

\section{ACKNOWLEDGEMENTS}

This research was supported in part by the Bio \& Medical Technology Development Program (NRF-2017M3A9E2056460) and the Basic Science Research Program (NRF-2018R1A2B6003917) through the National Research Foundation of Korea (NRF) funded by the Ministry of Science and ICT (MSIP), and an Incheon National University Research Grant in 2017. This work was conducted during the research year of Chungbuk National University in 2018 .

\section{REFERENCES}

1. Sirotin YB, Das A (2010) Zooming in on mouse vision. Nat Neurosci 13:1045-1046.

2. Martin PR, Solomon SG (2011) Information processing in the primate visual system. J Physiol 589:29-31.

3. Salinas-Navarro M, Jiménez-López M, Valiente-Soriano FJ, Alarcón-Martínez L, Avilés-Trigueros M, Mayor S, Holmes T, Lund RD, Villegas-Pérez MP, Vidal-Sanz M (2009) Retinal ganglion cell population in adult albino and pigmented mice: a computerized analysis of the entire population and its spatial distribution. Vision Res 49:637-647.

4. Sun W, Li N, He S (2002) Large-scale morphological survey of mouse retinal ganglion cells. J Comp Neurol 451:115-126.

5. Völgyi B, Chheda S, Bloomfield SA (2009) Tracer coupling patterns of the ganglion cell subtypes in the mouse retina. J Comp Neurol 512:664-687.

6. Baden T, Berens P, Franke K, Román Rosón M, Bethge M, Euler T (2016) The functional diversity of retinal ganglion cells in the mouse. Nature 529:345-350.

7. Jouty J, Hilgen G, Sernagor E, Hennig MH (2018) Non-para- metric physiological classification of retinal ganglion cells in the mouse retina. Front Cell Neurosci 12:481.

8. Román Rosón M, Bauer Y, Kotkat AH, Berens P, Euler T, Busse L (2019) Mouse dLGN receives functional input from a diverse population of retinal ganglion cells with limited convergence. Neuron 102:462-476.e8.

9. Krieger B, Qiao M, Rousso DL, Sanes JR, Meister M (2017) Four alpha ganglion cell types in mouse retina: function, structure, and molecular signatures. PLoS One 12:e0180091.

10. Rousso DL, Qiao M, Kagan RD, Yamagata M, Palmiter RD, Sanes JR (2016) Two pairs of ON and OFF retinal ganglion cells are defined by intersectional patterns of transcription factor expression. Cell Rep 15:1930-1944.

11. Antinucci P, Suleyman O, Monfries C, Hindges R (2016) Neural mechanisms generating orientation selectivity in the retina. Curr Biol 26:1802-1815.

12. Dhande OS, Stafford BK, Franke K, El-Danaf R, Percival KA, Phan AH, Li P, Hansen BJ, Nguyen PL, Berens P, Taylor WR, Callaway E, Euler T, Huberman AD (2019) Molecular fingerprinting of on-off direction-selective retinal ganglion cells across species and relevance to primate visual circuits. J Neurosci 39:78-95.

13. Jacoby J, Schwartz GW (2017) Three small-receptive-field ganglion cells in the mouse retina are distinctly tuned to size, speed, and object motion. J Neurosci 37:610-625.

14. Zhang Y, Kim IJ, Sanes JR, Meister M (2012) The most numerous ganglion cell type of the mouse retina is a selective feature detector. Proc Natl Acad Sci U S A 109:E2391-E2398.

15. Chichilnisky EJ, Baylor DA (1999) Receptive-field microstructure of blue-yellow ganglion cells in primate retina. Nat Neurosci 2:889-893.

16. Joesch M, Meister M (2016) A neuronal circuit for colour vision based on rod-cone opponency. Nature 532:236-239.

17. Chander D, Chichilnisky EJ (2001) Adaptation to temporal contrast in primate and salamander retina. J Neurosci 21:9904-9916.

18. Coombs JL, Van Der List D, Chalupa LM (2007) Morphological properties of mouse retinal ganglion cells during postnatal development. J Comp Neurol 503:803-814.

19. Tikidji-Hamburyan A, Reinhard K, Seitter H, Hovhannisyan A, Procyk CA, Allen AE, Schenk M, Lucas RJ, Münch TA (2015) Retinal output changes qualitatively with every change in ambient illuminance. Nat Neurosci 18:66-74.

20. Schmidt TM, Alam NM, Chen S, Kofuji P, Li W, Prusky GT, Hattar S (2014) A role for melanopsin in alpha retinal ganglion cells and contrast detection. Neuron 82:781-788.

21. Kuo SP, Schwartz GW, Rieke F (2016) Nonlinear spatiotem- 
poral integration by electrical and chemical synapses in the retina. Neuron 90:320-332.

22. Roska B, Nemeth E, Orzo L, Werblin FS (2000) Three levels of lateral inhibition: a space-time study of the retina of the tiger salamander. J Neurosci 20:1941-1951.

23. Sabharwal J, Seilheimer RL, Cowan CS, Wu SM (2016) The ON crossover circuitry shapes spatiotemporal profile in the center and surround of mouse OFF retinal ganglion cells. Front Neural Circuits 10:106.

24. Pillow JW, Shlens J, Paninski L, Sher A, Litke AM, Chichilnisky EJ, Simoncelli EP (2008) Spatio-temporal correlations and visual signalling in a complete neuronal population. Nature 454:995-999.

25. Schwartz O, Pillow JW, Rust NC, Simoncelli EP (2006) Spiketriggered neural characterization. J Vis 6:484-507.

26. Anishchenko A, Greschner M, Elstrott J, Sher A, Litke AM, Feller MB, Chichilnisky EJ (2010) Receptive field mosaics of retinal ganglion cells are established without visual experience. J Neurophysiol 103:1856-1864.

27. Chichilnisky EJ, Kalmar RS (2002) Functional asymmetries in $\mathrm{ON}$ and OFF ganglion cells of primate retina. J Neurosci 22:2737-2747.

28. Devries SH, Baylor DA (1997) Mosaic arrangement of ganglion cell receptive fields in rabbit retina. J Neurophysiol 78:2048-2060.

29. Gauthier JL, Field GD, Sher A, Greschner M, Shlens J, Litke AM, Chichilnisky EJ (2009) Receptive fields in primate retina are coordinated to sample visual space more uniformly. PLoS Biol 7:e1000063.

30. Gauthier JL, Field GD, Sher A, Shlens J, Greschner M, Litke AM, Chichilnisky EJ (2009) Uniform signal redundancy of parasol and midget ganglion cells in primate retina. J Neurosci 29:4675-4680.

31. Greschner M, Field GD, Li PH, Schiff ML, Gauthier JL, Ahn D, Sher A, Litke AM, Chichilnisky EJ (2014) A polyaxonal amacrine cell population in the primate retina. J Neurosci 34:3597-3606.

32. Brown SP, He S, Masland RH (2000) Receptive field microstructure and dendritic geometry of retinal ganglion cells. Neuron 27:371-383.

33. Field GD, Chichilnisky EJ (2007) Information processing in the primate retina: circuitry and coding. Annu Rev Neurosci 30:1-30.

34. Johnson KP, Zhao L, Kerschensteiner D (2018) A pixel-encoder retinal ganglion cell with spatially offset excitatory and inhibitory receptive fields. Cell Rep 22:1462-1472.

35. Samengo I, Gollisch T (2013) Spike-triggered covariance: geometric proof, symmetry properties, and extension beyond Gaussian stimuli. J Comput Neurosci 34:137-161.

36. Sandler RA, Marmarelis VZ (2015) Understanding spiketriggered covariance using Wiener theory for receptive field identification. J Vis 15:16.

37. Pillow JW, Simoncelli EP (2006) Dimensionality reduction in neural models: an information-theoretic generalization of spike-triggered average and covariance analysis. J Vis 6:414428.

38. Saleem AB, Krapp HG, Schultz SR (2008) Receptive field characterization by spike-triggered independent component analysis. J Vis 8:2.1-16.

39. Fairhall AL, Burlingame CA, Narasimhan R, Harris RA, Puchalla JL, Berry MJ 2nd (2006) Selectivity for multiple stimulus features in retinal ganglion cells. J Neurophysiol 96:27242738.

40. Gollisch T (2013) Features and functions of nonlinear spatial integration by retinal ganglion cells. J Physiol Paris 107:338348.

41. Gollisch T, Meister M (2008) Modeling convergent ON and OFF pathways in the early visual system. Biol Cybern 99:263278.

42. Liu JK, Gollisch T (2015) Spike-triggered covariance analysis reveals phenomenological diversity of contrast adaptation in the retina. PLoS Comput Biol 11:e1004425.

43. Ahn KN, Ahn JY, Kim JH, Cho K, Koo KI, Senok SS, Goo YS (2015) Effect of stimulus waveform of biphasic current pulse on retinal ganglion cell responses in retinal degeneration ( $\mathrm{rd} 1$ ) mice. Korean J Physiol Pharmacol 19:167-175.

44. Reinhard K, Tikidji-Hamburyan A, Seitter H, Idrees S, Mutter M, Benkner B, Münch TA (2014) Step-by-step instructions for retina recordings with perforated multi electrode arrays. PLoS One 9:e106148.

45. Lewicki MS (1998) A review of methods for spike sorting: the detection and classification of neural action potentials. Network 9:R53-R78.

46. Brainard DH (1997) The psychophysics toolbox. Spat Vis 10:433-436.

47. Pelli DG (1997) The VideoToolbox software for visual psychophysics: transforming numbers into movies. Spat Vis 10:437-442.

48. Wang YV, Weick M, Demb JB (2011) Spectral and temporal sensitivity of cone-mediated responses in mouse retinal ganglion cells. J Neurosci 31:7670-7681.

49. Simoncelli E, Pillow JW, Paninski L, Schwartz O (2004) Characterization of neural responses with stochastic stimuli. In: The new cognitive neurosciences III (Gazzaniga MS, ed), 3rd 
ed. pp 327-338. MIT Press, Cambridge.

50. Tachibana M, Okada T (1991) Release of endogenous excitatory amino acids from ON-type bipolar cells isolated from the goldfish retina. J Neurosci 11:2199-2208.

51. Slaughter MM, Miller RF (1983) Bipolar cells in the mudpuppy retina use an excitatory amino acid neurotransmitter. Nature 303:537-538.

52. Matsui K, Hosoi N, Tachibana M (1998) Excitatory synaptic transmission in the inner retina: paired recordings of bipolar cells and neurons of the ganglion cell layer. J Neurosci 18:4500-4510.

53. Bloomfield SA, Xin D (2000) Surround inhibition of mammalian AII amacrine cells is generated in the proximal retina. J Physiol 523 Pt 3:771-783.

54. Balasubramanian R, Gan L (2014) Development of retinal amacrine cells and their dendritic stratification. Curr Ophthalmol Rep 2:100-106.

55. Masland RH (2012) The tasks of amacrine cells. Vis Neurosci 29:3-9.

56. Cantrell DR, Cang J, Troy JB, Liu X (2010) Non-centered spike-triggered covariance analysis reveals neurotrophin-3 as a developmental regulator of receptive field properties of ON-OFF retinal ganglion cells. PLoS Comput Biol 6:e1000967.

57. Liu JK, Schreyer HM, Onken A, Rozenblit F, Khani MH, Krishnamoorthy V, Panzeri S, Gollisch T (2017) Inference of neuronal functional circuitry with spike-triggered nonnegative matrix factorization. Nat Commun 8:149.

58. Shi Q, Gupta P, Boukhvalova AK, Singer JH, Butts DA (2019) Functional characterization of retinal ganglion cells using tailored nonlinear modeling. Sci Rep 9:8713.

59. Bonin V, Histed MH, Yurgenson S, Reid RC (2011) Local diversity and fine-scale organization of receptive fields in mouse visual cortex. J Neurosci 31:18506-18521.

60. Nishimoto S, Gallant JL (2011) A three-dimensional spatiotemporal receptive field model explains responses of area MT neurons to naturalistic movies. J Neurosci 31:14551-14564.

61. Barabas P, Huang W, Chen H, Koehler CL, Howell G, John SW, Tian N, Rentería RC, Krizaj D (2011) Missing optomotor head-turning reflex in the DBA/2J mouse. Invest Ophthalmol Vis Sci 52:6766-6773.

62. Chen H, Zhao Y, Liu M, Feng L, Puyang Z, Yi J, Liang P, Zhang HF, Cang J, Troy JB, Liu X (2015) Progressive degeneration of retinal and superior collicular functions in mice with sustained ocular hypertension. Invest Ophthalmol Vis Sci 56:1971-1984.

63. Marc RE, Jones BW, Anderson JR, Kinard K, Marshak DW,
Wilson JH, Wensel T, Lucas RJ (2007) Neural reprogramming in retinal degeneration. Invest Ophthalmol Vis Sci 48:33643371.

64. Jones BW, Pfeiffer RL, Ferrell WD, Watt CB, Marmor M, Marc RE (2016) Retinal remodeling in human retinitis pigmentosa. Exp Eye Res 150:149-165.

65. Goo YS, Park DJ, Ahn JR, Senok SS (2016) Spontaneous oscillatory rhythms in the degenerating mouse retina modulate retinal ganglion cell responses to electrical stimulation. Front Cell Neurosci 9:512.

66. Trenholm S, Awatramani GB (2015) Origins of spontaneous activity in the degenerating retina. Front Cell Neurosci 9:277.

67. Ahn SM, Ahn J, Cha S, Yun C, Park TK, Goo YS, Kim SW (2019) Development of a post-vitrectomy injection of Nmethyl-N-nitrosourea as a localized retinal degeneration rabbit model. Exp Neurobiol 28:62-73.

68. Yu WQ, Grzywacz NM, Lee EJ, Field GD (2017) Cell typespecific changes in retinal ganglion cell function induced by rod death and cone reorganization in rats. J Neurophysiol 118:434-454.

69. Stingl K, Bartz-Schmidt KU, Besch D, Chee CK, Cottriall CL, Gekeler F, Groppe M, Jackson TL, MacLaren RE, Koitschev A, Kusnyerik A, Neffendorf J, Nemeth J, Naeem MA, Peters T, Ramsden JD, Sachs H, Simpson A, Singh MS, Wilhelm B, Wong D, Zrenner E (2015) Subretinal visual implant alpha IMS--clinical trial interim report. Vision Res 111:149-160.

70. Luo YH, da Cruz L (2016) The Argus $\left({ }^{\circledR}\right)$ II retinal prosthesis system. Prog Retin Eye Res 50:89-107.

71. Jalligampala A, Sekhar S, Zrenner E, Rathbun DL (2017) Optimal voltage stimulation parameters for network-mediated responses in wild type and $\mathrm{rd} 10$ mouse retinal ganglion cells. J Neural Eng 14:026004.

72. Haq W, Dietter J, Zrenner E (2018) Electrical activation of degenerated photoreceptors in blind mouse retina elicited network-mediated responses in different types of ganglion cells. Sci Rep 8:16998.

73. Im M, Werginz P, Fried SI (2018) Electric stimulus duration alters network-mediated responses depending on retinal ganglion cell type. J Neural Eng 15:036010.

74. Zeck GM, Xiao Q, Masland RH (2005) The spatial filtering properties of local edge detectors and brisk-sustained retinal ganglion cells. Eur J Neurosci 22:2016-2026.

75. Esler TB, Maturana MI, Kerr RR, Grayden DB, Burkitt AN, Meffin H (2018) Biophysical basis of the linear electrical receptive fields of retinal ganglion cells. J Neural Eng 15:055001.

76. Rathbun DL, Ghorbani N, Shabani H, Zrenner E, Hosseinzadeh Z (2018) Spike-triggered average electrical stimuli 
as input filters for bionic vision-a perspective. J Neural Eng 15:063002.

77. Maturana MI, Apollo NV, Garrett DJ, Kameneva T, Cloherty
SL, Grayden DB, Burkitt AN, Ibbotson MR, Meffin H (2018) Electrical receptive fields of retinal ganglion cells: influence of presynaptic neurons. PLoS Comput Biol 14:e1005997. 